\title{
SUFFICIENT SECOND-ORDER OPTIMALITY CONDITIONS FOR AN ELLIPTIC OPTIMAL CONTROL PROBLEM WITH POINTWISE CONTROL-STATE CONSTRAINTS
}

\author{
A. RÖSCH * AND F. TRÖLTZSCH ${ }^{\dagger}$
}

\begin{abstract}
An optimal control problem for an semilinear elliptic equation is investigated, where pointwise constraints are given on the control and the state. The state constraints are of mixed (bottleneck) type, where associated Lagrange multipliers can assumed to be bounded and measurable functions. Based on this property, a second-order sufficient optimality condition is established that considers strongly active constraints.
\end{abstract}

Keywords: Optimal control, elliptic differential equation, sufficient second-order optimality condition, pointwise mixed control-state constraints

AMS subject classification: $49 \mathrm{~K} 20,90 \mathrm{C} 48$

1. Introduction. In this paper we consider the optimal control problem to minimize

$$
F(y, u)=\int_{\Omega} f(x, y(x)) d x+\int_{\Gamma} g(x, y(x), u(x)) d s(x)
$$

subject to the state equations

$$
\begin{aligned}
A y+y & =0 & & \text { in } \Omega \\
\partial_{n_{A}} y & =b(x, y, u) & & \text { on } \Gamma,
\end{aligned}
$$

the control constraints

$$
0 \leq u(x) \text { for } x \in \Gamma,
$$

and to the mixed control-state constraints

$$
c(x) \leq u(x)+\gamma(x) y(x) \quad \text { for } x \in \Gamma .
$$

The main task of our paper is to establish second-order sufficient optimality conditions that are close to the associated necessary ones. For control-constrained problems, this issue was discussed quite completely in literature for semilinear elliptic and parabolic equations. We mention Bonnans [4], Casas, Tröltzsch, and Unger [9], Goldberg and Tröltzsch [12], Heinkenschloss and Tröltzsch [13].

The main difficulty in our problem is the presence of the pointwise control-state constraint $c(x) \leq u(x)+\gamma(x) y(x)$ in (1.4). If pointwise state contraints are given, then the theory of sufficient second-order conditions is faced with specific difficulties that are still far from being solved. In particular, these problems arise for pointwise state constraints of the type $c(x) \leq y(x)$. Here, the Lagrange multipliers associated with the state constraints are Borel measures so that the associated adjoint state exhibits low regularity, cf. Casas [5], [6] or Alibert and Raymond [1]. This fact causes specific difficulties in the discussion for second-order sufficient optimality conditions. We refer

*Johann Radon Institute for Computational and Applied Mathematics (RICAM), Austrian Academy of Sciences, Altenbergerstraße 69, A-4040 Linz, Austria, arnd.roesch@oeaw.ac.at

${ }^{\dagger}$ Technische Universität Berlin, Fakultät II - Mathematik und Naturwissenschaften, Str. des 17. Juni 136, D-10623 Berlin, Germany, troeltzsch@math.tu-berlin.de 
to Casas, Tröltzsch, and Unger [10] and Raymond and Tröltzsch [15] or to Casas and Mateos [7], who consider the case of finitely many state constraints.

In our problem (1.1)-(1.4), the situation is slightly simpler, since the constraint (1.4) is a mixed control-state constraint of bottleneck type. In the associated parabolic case, the Lagrange multipliers are more regular. They can assumed to be bounded and measurable functions, see Bergounioux and Tröltzsch [3], Arada and Raymond [2]. The existence of bounded and measurable Lagrange multipliers for linear-quadratic elliptic optimal control problems is proved in Tröltzsch [21]. The semilinear elliptic case is investigated in Rösch and Tröltzsch [16].

Higher regularity of the multipliers is the main advantage enabling us to establish second-order conditions. The second-order conditions should require minimal assumptions, i.e. they should be as close as possible to associated necessary conditions. Usually, this task is accomplished by considering strongly active sets (see [11] for control-constrained optimal control of ordinary differential equations). Here, we apply this technique to our case of mixed constraints. The analysis shows that this is not an easy task. It indicates that pointwise state constraints of more general type will rise even more difficult techniques.

Our paper extends the results of [17], [18] where second-order conditions are derived for a weakly singular integral state equation and for parabolic equations, respectively. Let us shortly sketch the main difference between these papers and our new discussion: In that papers, the proof of sufficiency is based on the nonnegativity of some inverse operators related to the Fréchet derivative of the control-to-state mapping. It is this nonnegativity that cannot in general be expected for elliptic problems. Therefore, here we abstain from such an assumption. We only require a solvability property of an auxiliary elliptic problem. Based on similarly weak assumptions, also the regularity of Lagrange multipliers has been shown in [16],[21].

Moreover, in our paper the definition of strongly active sets associated with the mixed constraints is is more natural way than the one in [17],[18].

Let us remark that the inequality constraints in our problem differ from the inequality constraints considered in [17],[18], where $u \leq c+\gamma y$ is investigated instead of (1.4). It would be easy to adapt our theory to the inequality constraints in [17],[18]. However, even for parabolic problems, the methods in [17],[18] cannot be applied to the inequality constraints (1.3) and (1.4): Formula (3.6) of [18] states that $f \geq 0$ implies $u \geq 0$. This is no longer true. The last implication in the proof of that formula is that $f \geq 0$ and $v \geq 0$ imply $u=f+\gamma v \geq 0$. Our inequality (1.4) leads now to the expression $u=f-\gamma v$ that may admit any sign.

The paper is organized as follows: In Section 2 we formulate first- and secondorder optimality conditions and state the main result. Section 3 contains auxiliary results. The proof that our second-order conditions are sufficient for local optimality is presented in Section 4.

In the paper, we use the following notations: $\mathrm{By} b^{\prime}(x, y, u)$ and $b^{\prime \prime}(x, y, u)$ we denote the gradient and the Hessian matrix of $b$ with respect to $(y, u)$ :

$$
b^{\prime}(x, y, u)=\left(\begin{array}{c}
b_{y}(x, y, u) \\
b_{u}(x, y, u)
\end{array}\right), \quad b^{\prime \prime}(x, y, u)=\left(\begin{array}{cc}
b_{y y}(x, y, u) & b_{y u}(x, y, u) \\
b_{y y}(x, y, u) & b_{u u}(x, y, u)
\end{array}\right) .
$$

Here, the notations $b_{y}(x, y, u)=D_{y} b(x, y, u)$ and $b_{y y}(x, y, u)=D_{y y} b(x, y, u)$ etc. are used for the partial derivatives. The norms $\left|b^{\prime}\right|,\left|b^{\prime \prime}\right|$ are defined by adding the absolute values of all entries of $b^{\prime}$ and $b^{\prime \prime}$, respectively. By $\partial_{n_{A}}$ we denote the co-normal derivative. 
We adapt the following assumptions from [9]:

(A1) For each $x \in \Omega$ or $\Gamma$ repectively, the functions $f=f(x, y), g=g(x, y, u)$, and $b=b(x, y, u)$ are of class $C^{2}$ with respect to $(y, u)$. For fixed $(y, u)$ they are Lebesgue measurable with respect to $x \in \Omega$ or $x \in \Gamma$, respectively.

(A2) In this assumption, fixed parameters $p>N-1$ and $s, r$ are used that depend on the dimension $N$ of the domain $\Omega$. Roughly speaking, we have $\left.y\right|_{\Gamma} \in L^{s}(\Gamma)$ and $y \in L^{r}(\Omega)$ in the linearized system associated to (1.2). As usual, $r^{\prime}$ and $s^{\prime}$ denote mutually conjugate numbers. For instance, $s^{\prime}$ is defined by $1 / s^{\prime}+1 / s=1$.

For all $M>0$, there are constants $C_{M}>0$, functions $\psi_{f}^{M} \in L^{(r / 2)^{\prime}}(\Omega)$, $\psi_{f}^{M, 1} \in L^{(s / 2)^{\prime}}(\Gamma), \psi_{f}^{M, 2} \in L^{(s / 2)^{\prime}}(\Gamma), \psi_{f}^{M, 3} \in L^{\infty}(\Gamma)$, and a continuous, monotone increasing function $\eta \in C\left(\mathbb{R}^{+} \cup\{0\}\right)$ with $\eta(0)=0$ such that

(i) $b(., 0,0) \in L^{p}(\Gamma)$, for some $p>N-1$,

$$
\begin{array}{cl} 
& b_{y}(x, y, u) \leq 0 \quad \text { for a.e. } x \in \Gamma, \forall(y, u) \in \mathbb{R}^{2}, \\
& \left|b^{\prime}(x, y, u)\right|+\left|b^{\prime \prime}(x, y, u)\right| \leq C_{M}, \\
& \left|b^{\prime \prime}\left(x, y_{1}, u_{1}\right)-b^{\prime \prime}\left(x, y_{2}, u_{2}\right)\right| \leq C_{M} \eta\left(\left|y_{1}-y_{2}\right|+\left|u_{1}-u_{2}\right|\right) \\
& \text { for almost all } x \in \Gamma \text { and all }|y|,|u|,\left|y_{i}\right|,\left|u_{i}\right| \leq M, i=1,2 . \\
\text { (ii) }) & f(., 0) \in L^{1}(\Omega), f_{y}(., 0) \in L^{r^{\prime}}(\Omega), f_{y y}(., 0) \in L^{(r / 2)^{\prime}}(\Omega) \text { and } \\
& \left|f_{y y}\left(x, y_{1}\right)-f_{y y}\left(x, y_{2}\right)\right| \leq \psi_{f}^{M}(x) \eta\left(\left|y_{1}-y_{2}\right|\right) \\
& \text { for almost all } x \in \Omega \text { and all }\left|y_{i}\right| \leq M, i=1,2 . \\
\text { (iii) } & g(., 0,0) \in L^{1}(\Gamma), g_{y}(., 0,0) \in L^{s^{\prime}}(\Gamma), g_{u} \in L^{2}(\Gamma), g_{y y}(., 0,0) \\
& \in L^{(s / 2)^{\prime}}(\Gamma), g_{y u}(., 0,0) \in L^{2(s / 2)^{\prime}}(\Gamma), g_{u u}(., 0,0) \in L^{\infty}(\Gamma), \text { and } \\
& \left|g_{y y}\left(x, u_{1}, y_{1}\right)-g_{y y}\left(x, u_{2}, y_{2}\right)\right| \leq \psi_{f}^{M, 1}(x) \eta\left(\left|y_{1}-y_{2}\right|+\left|u_{1}-u_{2}\right|\right) \\
& \left|g_{y u}\left(x, u_{1}, y_{1}\right)-g_{y u}\left(x, u_{2}, y_{2}\right)\right| \leq \psi_{f}^{M, 2}(x) \eta\left(\left|y_{1}-y_{2}\right|+\left|u_{1}-u_{2}\right|\right) \\
& \left|g_{y u}\left(x, u_{1}, y_{1}\right)-g_{y u}\left(x, u_{2}, y_{2}\right)\right| \leq \psi_{f}^{M, 3}(x) \eta\left(\left|y_{1}-y_{2}\right|+\left|u_{1}-u_{2}\right|\right)
\end{array}
$$

for almost all $x \in \Omega$ and all $\left|y_{i}\right| \leq M,\left|u_{i}\right| \leq M, i=1,2$.

Other estimates of $b, f, g$ and their first derivatives can be derived from (A1), (A2) by the mean value theorem.

(A3) We assume that $c, \gamma \in C(\Gamma)$, and $\gamma(x) \geq 0 \quad \forall x \in \Gamma$.

(A4) The domain $\Omega \subset \mathbb{R}^{N}$ is bounded and has a Lipschitz boundary $\Gamma$. The Lebesgue surface measure induced on $\Gamma$ is denoted by $d s(x)$. The elliptic operator $A$ is defined by

$$
A y(x)=-\sum_{i, j=1}^{m} D_{i}\left(a_{i j}(x) D_{j} y(x)\right)
$$

where $a_{i j} \in L^{\infty}(\Omega)$ satisfy, for some positive $m_{0}$, the condition of uniform ellipticity

$$
\sum_{i, j=1}^{m} a_{i j}(x) \xi_{i} \xi_{j} \geq m_{0}|\xi|^{2}
$$

2. First- and second-order optimality conditions. The control is looked for in the space $U=L^{\infty}(\Gamma)$, while the state is defined as weak solution of (1.2) in the 
state space $Y=C(\bar{\Omega}) \cap H^{1}(\Omega)$ by

$$
\int_{\Omega}\left(\sum_{i, j=1}^{m} a_{i j} D_{j} y D_{i} v+y v\right) d x=\int_{\Gamma} b(., y, u) v d s(x) \quad \forall v \in H^{1}(\Omega) .
$$

We endow $Y$ with the norm $\|y\|_{Y}=\|y\|_{C(\bar{\Omega})}+\|y\|_{H^{1}(\Omega)}$. It can be shown that the equation (1.2) admits for each $u \in L^{\infty}(\Gamma)$ a unique weak solution $y=y(u) \in Y$, see [8]. Moreover, Casas and Tröltzsch [8] have proved that the solution mapping $G: u \mapsto y$ from $L^{\infty}(\Omega)$ into $Y$ is of class $C^{2}$.

In this paper, we discuss sufficient conditions for a local minimum. Therefore, we investigate a candidate $\bar{u}$ for the local optimum and an $\varepsilon$-neighbourhood of this point:

$$
B_{\varepsilon}(\bar{u})=\left\{u \in L^{\infty}(\Gamma):\|u-\bar{u}\|_{L^{\infty}(\Gamma)}<\varepsilon\right\} .
$$

For any fixed $\varepsilon>0$ and arbitrary $\bar{u} \in U$, it holds with some constant $M=M(\varepsilon)$

$$
\|y(u)\|_{Y} \leq M \quad \forall y \in B_{\varepsilon}(\bar{u}) .
$$

The boundary values of $y$ are of particular importance for us. Thus we define the mapping $S: L^{\infty}(\Gamma) \rightarrow C(\bar{\Gamma})$ with $S=\tau G$ that assigns to $u$ the boundary values of $y$. Here, $\tau$ denote the trace operator. Clearly, the Fréchet differentiability of the operator $G$ transfers to $S$. The application of $S^{\prime}(\bar{u})$ to an element $h \in U$ is given by the boundary values of the solution $z$ of the elliptic problem

$$
\begin{aligned}
A z+z & =0 & & \text { in } \Omega \\
\partial_{n_{A}} z-\bar{b}_{y} z & =\bar{b}_{u} h & & \text { on } \Gamma,
\end{aligned}
$$

i.e. $S^{\prime}(\bar{u}) h=\left.z\right|_{\Gamma}$. Here we have used the abbreviations $\bar{b}_{y}=b_{y}(x, \bar{y}(x), \bar{u}(x))$ and $\bar{b}_{u}=b_{u}(x, \bar{y}(x), \bar{u}(x))$. The operator $S^{\prime}(\bar{u})$ extends to a linear continuous operator in $\mathcal{L}\left(L^{2}(\Gamma)\right)$. From now on, we consider $S^{\prime}(\bar{u})$ in this way. For the remainder term in the first order Taylor expansion of $y(\bar{u}+h)$, we obtain the property

$$
\frac{\|y(\bar{u}+h)-y(\bar{u})-z(\bar{u}, h)\|_{L^{2}(\Gamma)}}{\|h\|_{L^{2}(\Gamma)}} \rightarrow 0 \quad \text { as }\|h\|_{L^{\infty}(\Gamma)} \rightarrow 0
$$

using a known result of Maurer [14].

Next, we introduce the $L^{2}$-adjoint operator $S^{\prime}(\bar{u})^{*} \in \mathcal{L}\left(L^{2}(\Gamma)\right)$. This operator is given by $S^{\prime}(\bar{u})^{*} \mu=\left.\varphi\right|_{\Gamma}$, where $\varphi$ is the solution of the elliptic problem

$$
\begin{aligned}
A^{*} \varphi+\varphi & =0 & & \text { in } \Omega \\
\partial_{n_{A^{*}}} \varphi-\bar{b}_{y} \varphi & =\bar{b}_{u} \mu & & \text { on } \Gamma
\end{aligned}
$$

and $A^{*}$ is the formal adjoint operator to $A$. In all what follows, let $(\bar{y}, \bar{u})$ be a locally optimal reference solution of (1.1)-(1.4). Let us set up the associated first-order necessary optimality conditions in form of a Karush-Kuhn-Tucker type theorem. To this aim, we introduce the Lagrange functional $L: Y \times L^{\infty}(\Gamma) \times Y \times L^{\infty}(\Gamma)^{2} \rightarrow \mathbb{R}$,

$$
\begin{gathered}
L\left(y, u, p, \mu_{1}, \mu_{2}\right)= \\
F(y, u)+\int_{\Omega}\left(\sum_{i, j=1}^{m} a_{i j} D_{j} y D_{i} p+y p\right) d x-\int_{\Gamma} b p d s(x) \\
-\int_{\Gamma} \mu_{1} u d s(x)-\int_{\Gamma}(u+\gamma y-c) \mu_{2} d s(x) .
\end{gathered}
$$


Let us comment on this choice for $L$. The elliptic quation (1.2) is considered in $Y$, while the inequality constraints $(1.3)$ are posed in $L^{\infty}(\Gamma)$. Knowing the general Karush-Kuhn-Tucker theory in Banach spaces, one expects associated Lagrange multipliers $p \in Y^{*}$ and $\mu_{i} \in\left(L^{\infty}(\Gamma)\right)^{*}$, together with a related quite complicated Lagrange functional. In contrast to this, special techniques for optimal control problems of bottleneck type have shown that, under natural assumptions, the Lagrange multipliers can be expressed by regular functions, i.e. $p \in Y$ and $\mu_{i} \in L^{\infty}(\Gamma)$, we refer to Tröltzsch [21], Rösch and Tröltzsch [16]. This well known advantage of bottleneck type problems is our key idea to establish special second-order sufficient optimality conditions, which can hardly be expected for $\mu_{i} \in\left(L^{\infty}(\Gamma)\right)^{*}$. The existence of such regular multipliers can be shown under a Slater type condition and the assumption $\gamma(x) \geq 0$. Here, the nonnegativity of $\gamma$ plays a crucial role.

Therefore, we are justified to assume that an adjoint state $\bar{p} \in Y$ and Lagrange multipliers $\bar{\mu}_{i} \in L^{\infty}(\Gamma)$ exist such that $\left(\bar{y}, \bar{u}, \bar{p}, \bar{\mu}_{1}, \bar{\mu}_{2}\right)$ satisfies the following first-order necessary optimality system (FON),

$$
(F O N) \begin{cases}D_{y} L\left(\bar{y}, \bar{u}, \bar{p}, \bar{\mu}_{1}, \bar{\mu}_{2}\right) & =0 \\ D_{u} L\left(\bar{y}, \bar{u}, \bar{p}, \bar{\mu}_{1}, \bar{\mu}_{2}\right) & =0 \\ \text { and for almost all } x \in \Gamma & \geq 0 \\ \bar{\mu}_{1}(x) & \geq 0 \\ \bar{\mu}_{2}(x) & =0 \\ \bar{u}(x) \bar{\mu}_{1}(x) & \\ (\bar{u}(x)+\gamma(x) \bar{y}(x)-c(x)) \bar{\mu}_{2}(x) & =0 .\end{cases}
$$

Note that the Lagrange multipliers may be not unique. The last two conditions of (FON) are the well-known complementary slackness conditions. They imply $\bar{\mu}_{1}(x)>$ $0 \Rightarrow \bar{u}(x)=0$ and $\bar{\mu}_{2}(x)>0 \Rightarrow c(x)=\bar{u}(x)+\gamma(x) \bar{y}(x)$. Let us express these optimality conditions also in terms of the partial differential equation. As it is well known, the first equation of $(\mathrm{FON})$ is equivalent to the adjoint equation

$$
\begin{aligned}
A^{*} \bar{p}+\bar{p} & =f_{y}(x, \bar{y}) & & \text { in } \Omega \\
\partial_{n_{A^{*}}} \bar{p}-b_{y}(x, \bar{y}, \bar{u}) \bar{p} & =g_{y}(x, \bar{y}, \bar{u})-\gamma \bar{\mu}_{2} & & \text { on } \Gamma .
\end{aligned}
$$

The second equation of $(\mathrm{FON})$ is equivalent to

$$
g_{u}(x, \bar{y}, \bar{u})+b_{u}(x, \bar{y}, \bar{u}) \bar{p}-\bar{\mu}_{1}-\bar{\mu}_{2}=0 .
$$

Next, we discuss a sufficient second-order optimality condition (SSC). For this purpose, following Dontchev et al. [11], we define strongly active sets and the associated critical subspace. Assume that $\left(\bar{y}, \bar{u}, \bar{p}, \bar{\mu}_{1}, \bar{\mu}_{2}\right)$ fulfils (FON).

Definition 2.1. Let $\delta_{1}, \delta_{2}>0$ be real numbers and $\bar{\mu}_{1}, \bar{\mu}_{2} \in L^{\infty}(\Gamma)$ be Lagrange multipliers introduced in (FON). The sets

$$
\begin{aligned}
& A_{1}\left(\delta_{1}\right):=\left\{x \in \Gamma: \bar{\mu}_{1}(x) \geq \delta_{1}\right\}, \\
& A_{2}\left(\delta_{2}\right):=\left\{x \in \Gamma \backslash A_{1}\left(\delta_{1}\right): \bar{\mu}_{2}(x) \geq \delta_{2}\right\}
\end{aligned}
$$

are called strongly active sets.

All further arguments hold true for an arbitrary choice of $\delta_{1}$ and $\delta_{2}$. Later, these numbers will be chosen such that a second-order sufficient optimality condition is 
satisfied. To shorten the notation, we will drop the dependence of the active sets on these parameters in the proofs, but we will use the detailed notation for the statements of the main results.

Definition 2.2. We say that $(y, u) \in C(\bar{\Omega}) \times L^{\infty}(\Gamma)$ belongs to the critical subspace, if

$$
\begin{aligned}
& u=0 \quad \text { on } A_{1}\left(\delta_{1}\right) \text {, } \\
& u+\left.\gamma y\right|_{\Gamma}=0 \quad \text { on } A_{2}\left(\delta_{2}\right) \text {, }
\end{aligned}
$$

and

$$
\begin{aligned}
A y+y & =0 & & \text { in } \Omega \\
\partial_{n_{A}} y-\bar{b}_{y} y & =\bar{b}_{u} u & & \text { on } \Gamma .
\end{aligned}
$$

Notice that (2.10) implies $\left.y\right|_{\Gamma}=S^{\prime}(\bar{u}) u$. This assumption is stronger than really needed. A smaller critical convex cone is discussed at the end of the paper.

Before we formulate the second-order sufficient optimality condition, we mention for convenience the explicit expression of $L_{(u, y)}^{\prime \prime}\left(\bar{y}, \bar{u}, \bar{p}, \bar{\mu}_{1}, \bar{\mu}_{2}\right)\left[h_{y}, h_{u}\right]^{2}$ :

$$
\begin{aligned}
L_{(u, y)}^{\prime \prime}\left(\bar{y}, \bar{u}, \bar{p}, \bar{\mu}_{1}, \bar{\mu}_{2}\right)\left[h_{y}, h_{u}\right]^{2} & =\int_{\Omega} f_{y y} h_{y}^{2} d x+\int_{\Gamma}\left(g_{y y} h_{y}^{2}+2 g_{y u} h_{y} h_{u}+g_{u u} h_{u}^{2}\right) d s(x) \\
& +\int_{\Gamma}\left(\bar{b}_{y y} h_{y}^{2}+2 \bar{b}_{y u} h_{y} h_{u}+\bar{b}_{u u} h_{u}^{2}\right) \bar{p} d s(x) .
\end{aligned}
$$

Here, $h_{y} \in C(\bar{\Omega}), h_{u} \in L^{\infty}(\Gamma)$ denote arbitrary increments of $y$ and $u$, respectively. Now we state the second-order sufficient condition.

(SSC): There exist positive numbers $\delta, \delta_{1}, \delta_{2}$ such that the definiteness condition

$$
L_{(u, y)}^{\prime \prime}\left(\bar{y}, \bar{u}, \bar{p}, \bar{\mu}_{1}, \bar{\mu}_{2}\right)\left[h_{y}, h_{u}\right]^{2} \geq \delta\left\|h_{u}\right\|_{L^{2}(\Gamma)}^{2}
$$

holds true for all $\left(h_{y}, h_{u}\right)$ belonging to the critical subspace defined upon $\delta_{1}, \delta_{2}$.

In our further analysis, the boundary value problem

$$
\begin{aligned}
A v+v & =0 & & \text { in } \Omega \\
\partial_{n_{A}} v+\left(-\bar{b}_{y}+\chi_{A_{2}\left(\delta_{2}\right)} \bar{b}_{u} \gamma\right) v & =\phi & & \text { on } \Gamma
\end{aligned}
$$

plays a basic role. We require the following regularity assumption:

(R) For $\phi=0$, the problem (2.13) has only the trivial solution $v=0$.

For instance, this assumption is fulfilled, if

$$
-\bar{b}_{y}+\gamma \chi_{A_{2}\left(\delta_{2}\right)} \bar{b}_{u} \geq 0 \quad \text { a.e. on } \Gamma \text {. }
$$

Here $\chi_{A_{2}}$ denotes the characteristic function of the set $A_{2}\left(\delta_{2}\right)$. In particular, this condition is fulfilled if $\bar{b}_{u} \geq 0$ holds. Now, we state the main result of the paper.

TheOREM 2.3. (Second-order sufficiency) Assume that $\left(\bar{y}, \bar{u}, \bar{p}, \bar{\mu}_{1}, \bar{\mu}_{2}\right)$ fulfils the first-order optimality system (FON) and the regularity condition ( $R$ ) holds. If the second-order condition (SSC) is satisfied, then there exist $\delta_{s}>0$ and $\varepsilon>0$ such that the quadratic growth condition

$$
F(y, u)-F(\bar{y}, \bar{u}) \geq \delta_{s}\|u-\bar{u}\|_{L^{2}(\Gamma)}^{2}
$$

holds for all admissible pairs $(y, u)$ with $\|u-\bar{u}\|_{L^{\infty}(\Gamma)}<\varepsilon$. Therefore, $\bar{u}$ is a locally optimal control in the norm of $L^{\infty}(\Gamma)$.

The proof is carried out in Section 4 . 
3. Auxiliary results. Lemma 3.1. Let $\beta \in L^{\infty}(\Gamma)$ be a fixed function that is almost everywhere nonnegative. Then for all $\phi \in L^{p}(\Gamma)$ with $p>N-1$, the weak solution $v \in H^{1}(\Omega)$ of

$$
\begin{aligned}
A v+v & =0 & & \text { in } \Omega \\
\partial_{n_{A}} v+\beta(\cdot) v & =\phi & & \text { on } \Gamma
\end{aligned}
$$

belongs to $C(\bar{\Omega})$ and satisfies the estimate

$$
\|v\|_{C(\bar{\Omega})} \leq c_{p}\|\phi\|_{L^{p}(\Gamma)}
$$

with a positive constant $c_{p}$ that does not depend on $\phi$.

For this classical result, we refer to [6] and to the arguments in [1] concerning the case of Lipschitz domains.

The trace of $v \in H^{1}(\Omega)$ belongs for $N=2$ and any $r<\infty$ to $L^{r}(\Gamma)$ and for $N>2$ to $L^{\frac{2(N-1)}{N-2}}(\Gamma)$. Therefore, it belongs to $L^{2+s}(\Gamma)$ with $s=2 /(N-2)>0$ (arbitrary $s>0$ for $N=2$ ) so that the mapping $\phi \mapsto v$ is continuous from $L^{2}(\Gamma)$ to $L^{2+s}(\Gamma)$ and from $L^{p}(\Gamma)$ to $L^{\infty}(\Gamma)$, in particular also to $L^{p+s}(\Gamma)$. By classical interpolation, cf. Triebel [20], 1.18.7, Thm. 1, the mapping $\phi \mapsto v$ satisfies

$$
\|v\|_{L^{s+\delta}(\Gamma)} \leq c_{s}\|\phi\|_{L^{s}(\Gamma)} \quad \forall s \geq 2 .
$$

provided that $\beta(\cdot) \geq 0$. We can dispense with this sign condition on $\beta$, if a regularity condition is fulfilled. Therefore, we now consider equation (3.1) for arbitrary $\beta \in$ $L^{\infty}(\Gamma)$ and assume that the associated homogeneous equation (3.1) has only the trivial solution. Then the mapping $S_{\beta}: L^{2}(\Gamma) \rightarrow L^{2}(\Gamma)$ that assigns to $\phi$ the trace of the solution $v$ of (3.1) is well defined and continuous.

To verify this, we consider also the shifted equation

$$
\begin{aligned}
A v+v & =0 & & \text { in } \Omega \\
\partial_{n_{A}} v+\left(\|\beta\|_{L^{\infty}(\Gamma)}+\beta(\cdot)\right) v & =\phi & & \text { on } \Gamma .
\end{aligned}
$$

Clearly, the associated mapping $\tilde{S}_{\beta}: L^{2}(\Gamma) \rightarrow L^{2}(\Gamma), \tilde{S}_{\beta}: \phi \mapsto v$, is well defined and compact. By the Fredholm theory, it has only countably many eigenvalues. A number $\lambda \in \mathbb{R}$ is an eigenvalue of $\tilde{S}_{\beta}$, if in (3.4) the boundary condition

$$
\partial_{n_{A}} v+\left(\|\beta\|_{L^{\infty}(\Gamma)}+\beta(\cdot)\right) v=\lambda^{-1} v
$$

is satisfied with some nontrivial $v$. Obviously, we have a one-to-one correspondence between the eigenvalues of $\tilde{S}_{\beta}$ and those of $S_{\beta}$. The boundary condition (3.5) holds for nontrivial $v$ iff the condition $\partial_{n_{A}} v+\left(\|\beta\|_{L^{\infty}(\Gamma)}+\beta(\cdot)\right) v=\left(\lambda^{-1}-\|\beta\|_{L^{\infty}(\Gamma)}\right) v$ is fulfilled so that $1 /\left(\lambda^{-1}-\|\beta\|_{L^{\infty}(\Gamma)}\right)$ is eigenvalue of $S_{\beta}$.

In view of this, the assumption on the homogeneous equation (3.1) implies that (3.1) is uniquely solvable for all $\phi \in L^{2}(\Gamma)$ and that $S_{\beta}$ is continuous in $L^{2}(\Gamma)$.

In the following, we will use the short notation $\|v\|_{L^{2}(\Gamma)}$ instead of $\left\|\left.v\right|_{\tau^{\prime}}\right\|_{L^{2}(\Gamma)}$.

Lemma 3.2. Assume that the homogeneous equation (3.1) has only the trivial solution and that $\phi \in L^{\infty}(\Gamma)$ is given arbitrarily. Let $v \in H^{1}(\Omega)$ be the solution of (3.1). Then there exists a constant $c_{\beta}$ not depending on $\phi$ such that the following estimates hold true:

$$
\begin{aligned}
& \|v\|_{L^{2}(\Gamma)} \leq c_{\beta}\|\phi\|_{L^{2}(\Gamma)}, \\
& \|v\|_{C(\Gamma)} \leq c_{\beta}\|\phi\|_{L^{p}(\Gamma)} \text { for all } p>N-1, \\
& \|v\|_{L^{1}(\Gamma)} \leq c_{\beta}\|\phi\|_{L^{1}(\Gamma)} .
\end{aligned}
$$


Proof. (i) The first estimate is a simple consequence of the continuity of $S_{\beta}$ in $L^{2}(\Gamma)$. It is only stated for convenience.

(ii) The second inequality follows by bootstrapping: The solution $v$ solves $A v+v=$ 0 subject to the boundary condition

$$
\partial_{n_{A}} v=\phi-\beta(\cdot) v
$$

By (3.3) and the first estimate, we find with some $s>0$ and with some generic constant $c$

$$
\begin{aligned}
\|v\|_{L^{2+s}(\Gamma)} & \leq c\left(\|\phi\|_{L^{2}(\Gamma)}+\|\beta\|_{L^{\infty}(\Gamma)}\|v\|_{L^{2}(\Gamma)}\right) \\
& \leq c\|\phi\|_{L^{2}(\Gamma)} \leq c\|\phi\|_{L^{2+s}(\Gamma)} .
\end{aligned}
$$

Repeating this estimate, we get from the one in $L^{2+s}(\Gamma)$

$$
\begin{aligned}
\|v\|_{L^{2+2 s}(\Gamma)} & \leq c\left(\|\phi\|_{L^{2+s}(\Gamma)}+\|\beta\|_{L^{\infty}(\Gamma)}\|v\|_{L^{2+s}(\Gamma)}\right) \\
& \leq c\|\phi\|_{L^{2+s}(\Gamma)} \leq c\|\phi\|_{L^{2+2 s}(\Gamma)} .
\end{aligned}
$$

After finitely many steps, the estimate $\|v\|_{L^{p}(\Gamma)} \leq c\|\phi\|_{L^{p}(\Gamma)}$ can be derived for some $p>N-1$. Finally, again by (3.3), it follows

$$
\|v\|_{C(\Gamma)} \leq c_{p}\left(\|\phi\|_{L^{p}(\Gamma)}+\|\beta\|_{L^{\infty}(\Gamma)}\|v\|_{L^{p}(\Gamma)}\right) \leq c\|\phi\|_{L^{p}(\Gamma)} .
$$

(iii) To show the last estimate, we proceed by duality. The operator $S_{\beta}$ is selfadjoint. Moreover, roughly speaking, we have by (ii) that its restriction $S_{\beta, p}$ to $L^{p}(\Gamma)$ is continuous from $L^{p}(\Gamma)$ to $C(\Gamma)$. We can assume $p \geq 2$. The adjoint operator $S_{\beta, p}^{*}$ is continuous from $C(\Gamma)^{*}$ to $L^{p^{\prime}}(\Gamma)$, where $p^{\prime}$ is conjugate to $p$. Therefore, it is in particular continuous in $L^{1}(\Gamma)$. Finally, it can be shown that $S_{\beta, p}^{*} \phi=S_{\beta}^{*} \phi=S_{\beta} \phi$ for all $\phi \in L^{2}(\Gamma)$. This shows that $S_{\beta}$ is continuous in $L^{1}(\Gamma)$ so that the third estimate is true. These facts are explained more precise and slightly more detailed in [16].

We should remark that the third estimate is not surprising. For $\beta \geq 0$, it follows from the results by Casas [5] and Alibert and Raymond [1]: They have shown in this case that the boundary value problem (3.1) with given regular Borel measure $\phi$ admits a unique solution $v \in W^{1, \sigma}(\Omega)$ for all $\sigma<N /(N-1)$. Clearly, this implies the $L^{1}$-estimate. More or less, the result for arbitrary $\beta$ is a natural extension. We have presented these details for convenience of the reader.

As a corollary of the preceding Lemma, we obtain for $\beta:=-\bar{b}_{y}+\gamma \chi_{A_{2}} \bar{b}_{u}$ the following result:

Lemma 3.3. Suppose that the regularity condition $(R)$ is satisfied. Then, for all $\phi \in L^{2}(\Gamma)$, the boundary value problem

$$
\begin{aligned}
A v+v & =0 & & \text { in } \Omega \\
\partial_{n_{A}} v+\left(-\bar{b}_{y}+\chi_{A_{2}} \bar{b}_{u} \gamma\right) v & =\phi & & \text { in } \Gamma
\end{aligned}
$$

has a unique solution $v \in H^{1}(\Omega)$. Moreover, the estimate

$$
\|v\|_{L^{1}(\Gamma)} \leq c_{1}\|\phi\|_{L^{1}(\Gamma)}
$$

is fulfilled with some constant $c_{1}$ that does not depend on $\phi$.

To perform our analysis, we repeatedly need controls $u$ defined as follows:

$$
u(x)= \begin{cases}\phi(x) & \text { on } \Gamma \backslash A_{2}, \\ \phi(x)-\gamma(x)\left(S^{\prime}(\bar{u}) u\right)(x) & \text { on } A_{2} .\end{cases}
$$


The next lemma shows that this setting is correct:

Lemma 3.4. Assume that the regularity condition $(R)$ is fulfilled. Then, there is exactly one function $u \in L^{\infty}(\Gamma)$ that satisfies condition (3.9). Moreover, the estimates

$$
\begin{aligned}
\|u\|_{L^{1}(\Gamma)} & \leq c_{1}\|\phi\|_{L^{1}(\Gamma)} \\
\|u\|_{L^{2}(\Gamma)} & \leq c_{2}\|\phi\|_{L^{2}(\Gamma)} \\
\|u\|_{L^{\infty}(\Gamma)} & \leq c_{\infty}\|\phi\|_{L^{\infty}(\Gamma)}
\end{aligned}
$$

hold with certain constants $c_{1}, c_{2}, c_{\infty}$ that do not depend on $\phi$.

Proof. Suppose that $u \in L^{\infty}(\Gamma)$ satisfies (3.9). Put $v:=G^{\prime}(\bar{u}) u$. Then $v$ satisfies an elliptic problem with the boundary condition

$$
\partial_{n_{A}} v-\bar{b}_{y} v= \begin{cases}\bar{b}_{u} \phi & \text { on } \Gamma \backslash A_{2} \\ \bar{b}_{u}(\phi-\gamma v) & \text { on } A_{2}\end{cases}
$$

that is

$$
\partial_{n_{A}} v+\left(-\bar{b}_{y} v+\chi_{A_{2}} \bar{b}_{u} \gamma\right) v=\bar{b}_{u} \phi \quad \text { on } \Gamma \text {. }
$$

This is exactly the boundary condition of (3.7). Consequently, the solution $v$ is unique. Therefore, if $u$ satisfies (3.9), then $v=G^{\prime}(\bar{u}) u$ is unique, hence $u$ is unique, because of

$$
u= \begin{cases}\phi & \text { on } \Gamma \backslash A_{2} \\ \phi-\left.\gamma v\right|_{\Gamma} & \text { on } A_{2}\end{cases}
$$

On the other hand, starting from $\phi$, the solution $v$ of the elliptic equation with the boundary condition (3.14) is well defined, and the function $u$ given by (3.15) satisfies (3.9), since, by definition of $v, u=\left.S^{\prime}(\bar{u}) v\right|_{\Gamma}$.

The estimate (3.10) is obtained by Lemma 3.3. Estimate (3.11) follows by standard arguments. The Stampacchia method [19] delivers estimate (3.12).

To prove the main result, we later have to compare the reference pair $(\bar{y}, \bar{u})$ with another admissible pair $(y, u)$, where $y=G(u)$. In this case, we estimate the difference

$$
\left.y\right|_{\Gamma}-\left.\bar{y}\right|_{\Gamma}=S(u)-S(\bar{u})=S^{\prime}(\bar{u})(u-\bar{u})+r_{1}(\bar{u}, u-\bar{u})
$$

where $r_{1}$ stands for the associated first-order remainder term of $S$. In the following, if there is no risk of notational confusion, we denote for short the remainder $r_{1}(\bar{u}, u-\bar{u})$ and the derivative $S^{\prime}(\bar{u})$ by $r_{1}$ and $S^{\prime}$, respectively.

Before continuing our analysis of second-order sufficiency, let us discuss the main difficulties and our main ideas to resolve them. We start without the pointwise controlstate constraints. On $A_{1}$, we have $\bar{u}(x) \equiv 0$, hence $u-\bar{u} \geq 0$ on $A_{1}$. The associated term in the Lagrange functional can be estimated as

$$
\int_{A_{1}} \bar{\mu}_{1}(u-\bar{u}) d s(x) \geq \int_{A_{1}} \delta_{1}(u-\bar{u}) d s(x)=\delta_{1}\|u-\bar{u}\|_{L^{1}\left(A_{1}\right)} .
$$

In the proof of the sufficiency theorem, the $L^{1}$-norms on the right-hand side will compensate for the lack of coercivity, since (2.12) does not contribute to definiteness on $A_{1} \cup A_{2}$.

However, we cannot expect such a property for the mixed control-state constraints. It can happen that $\int_{A_{2}} \bar{\mu}_{2}(u+y-\bar{u}-\bar{y}) d s(x)=0$ although $\|u-\bar{u}\|_{L^{1}\left(A_{2}\right)}>0$ hold simultaneously. 
To overcome this difficulty, we represent $u$ in the form $u=u_{1}+u_{2}$. For the $u_{1}$ part we can prove an estimate similar to (3.17). The $u_{2}$-part stands for the additional margin of freedom that is caused by differing values of $u$ and $\bar{u}$ outside of $A_{2}$. This splitting is performed by

$$
\begin{array}{lll}
u_{1}=\bar{u}, & u_{2}=u-\bar{u} & \text { on } \Gamma \backslash A_{2}, \\
u_{2}=-\gamma\left(S^{\prime} u_{2}+r_{1}\right), & u_{1}=u-u_{2} & \text { on } A_{2} .
\end{array}
$$

The functions $u_{1}$ and $u_{2}$ are well defined. To see this, we write $u_{2}$ in the form

$$
u_{2}= \begin{cases}\phi & \text { on } \Gamma \backslash A_{2}, \\ \phi-\gamma S^{\prime} u_{2} & \text { on } A_{2},\end{cases}
$$

where $\phi=u-\bar{u}$ on $\Gamma \backslash A_{2}, \phi=\gamma r_{1}$ on $A_{2}$. Then $u_{2}$ is well defined by Lemma 3.4. Note that $S^{\prime}(\bar{u}) u_{2}=S^{\prime}(\bar{u})\left(\chi_{\Gamma \backslash A_{2}}(u-\bar{u})+\chi_{A_{2}} u_{2}\right)$. From (3.12) and (3.18) we get easily

$$
\left\|u_{2}\right\|_{L^{\infty}(\Gamma)} \leq c_{3}\left(\|u-\bar{u}\|_{L^{\infty}(\Gamma)}+\left\|r_{1}\right\|_{L^{\infty}(\Gamma)}\right) .
$$

The Fréchet differentiability of $S$ in $L^{\infty}(\Gamma)$ implies

$$
\left\|r_{1}\right\|_{L^{\infty}(\Gamma)} \leq\|u-\bar{u}\|_{L^{\infty}(\Gamma)}
$$

for a sufficiently small $\varepsilon$.

Therefore, it holds by $u_{1}=u-u_{2}$

$$
\begin{aligned}
\left\|u_{1}-\bar{u}\right\|_{L^{\infty}\left(A_{2}\right)} & \leq\|u-\bar{u}\|_{L^{\infty}\left(A_{2}\right)}+\left\|u_{2}\right\|_{L^{\infty}\left(A_{2}\right)} \\
& \leq c_{4}\|u-\bar{u}\|_{L^{\infty}(\Gamma) .}
\end{aligned}
$$

Lemma 3.5. Assume that $\left(\bar{y}, \bar{u}, \bar{p}, \bar{\mu}_{1}, \bar{\mu}_{2}\right)$ fulfils the first-order optimality system $(F O N)$ and the regularity condition $(R)$ holds. Then there exists a positive constant $c_{A}$ such that, for all $\varepsilon>0$ the estimates

$$
\begin{gathered}
\int_{\Gamma}(u-\bar{u}) \bar{\mu}_{1} d s(x) \geq \frac{\delta_{1}}{\varepsilon}\|u-\bar{u}\|_{L^{2}\left(A_{1}\left(\delta_{1}\right)\right)}^{2}, \\
\int_{\Gamma}(u-\bar{u}+\gamma(y-\bar{y})) \bar{\mu}_{2} d s(x) \geq c_{A} \cdot \frac{\delta_{2}}{\varepsilon}\left\|u_{1}-\bar{u}\right\|_{L^{2}\left(A_{2}\left(\delta_{2}\right)\right)}^{2}
\end{gathered}
$$

are valid for all admissible pairs $(u, y)$ satisfying $\|u-\bar{u}\|_{L^{\infty}(\Gamma)}<\varepsilon$.

Proof. (i) Because of (FON), $\bar{\mu}_{1}(x)>0$ can only hold where $\bar{u}(x)=0$. If $\bar{u}(x)>0$, then $\bar{\mu}_{1}(x)=0$. Moreover, $u$ is admissible, hence $u \geq 0$ and we have almost everywhere

$$
(u-\bar{u}) \bar{\mu}_{1} \geq 0
$$

Therefore we get by $(2.6)$

$$
\int_{\Gamma}(u-\bar{u}) \bar{\mu}_{1} d s(x) \geq \int_{A_{1}}(u-\bar{u}) \bar{\mu}_{1} d s(x) \geq \delta_{1}\|u-\bar{u}\|_{L^{1}\left(A_{1}\right)} .
$$

By our assumption, we have $\|u-\bar{u}\|_{L^{\infty}(\Gamma)}<\varepsilon$. In particular, this inequality includes $\|u-\bar{u}\|_{L^{\infty}\left(A_{1}\right)}<\varepsilon$. Consequently,

$$
\int_{\Gamma}(u-\bar{u}) \bar{\mu}_{1} d s(x) \geq \delta_{1}\|u-\bar{u}\|_{L^{1}\left(A_{1}\right)} \frac{\|u-\bar{u}\|_{L^{\infty}\left(A_{1}\right)}}{\varepsilon} \geq \frac{\delta_{1}}{\varepsilon}\|u-\bar{u}\|_{L^{2}\left(A_{1}\right)}^{2},
$$


and (3.20) is proven.

(ii) Next, we discuss the integral in (3.21). Because of (FON), $\bar{\mu}_{2}(x)>0$ can only hold where $\bar{u}(x)+\gamma(x) \bar{y}(x)=c(x)$. In addition, $(y, u)$ is admissible, hence in particular $c(x) \leq u(x)+\gamma(x) y(x)$. Therefore, we obtain almost everywhere

$$
(u-\bar{u}+\gamma(y-\bar{y})) \bar{\mu}_{2} \geq 0
$$

and

$$
\begin{aligned}
\int_{\Gamma}(u-\bar{u}+\gamma(y-\bar{y})) \bar{\mu}_{2} d s(x) & \geq \int_{A_{2}}(u-\bar{u}+\gamma(y-\bar{y})) \bar{\mu}_{2} d s(x) \\
& \geq \delta_{2}\|u-\bar{u}+\gamma(y-\bar{y})\|_{L^{1}\left(A_{2}\right)}
\end{aligned}
$$

by definition (2.7). Let us discuss this integral more detailed. Expressing $y-\bar{y}$ in terms of the controls by (3.16),

$$
u-\bar{u}+\gamma(y-\bar{y})=u-\bar{u}+\gamma\left(S^{\prime}(\bar{u})(u-\bar{u})+r_{1}\right)
$$

is found. Since $u=u_{1}+u_{2}$ and $u_{2}+\gamma S^{\prime} u_{2}+\gamma r_{1}=0$ on $A_{2}$ hold by definition (3.18), we find

$$
u+\gamma\left(S^{\prime} u+r_{1}\right)=u_{1}+u_{2}+\gamma S^{\prime} u_{1}+\gamma S^{\prime} u_{2}+\gamma r_{1}=u_{1}+\gamma S^{\prime} u_{1} .
$$

Consequently, (3.22) and (3.23) yield

$$
\begin{aligned}
\int_{\Gamma}(u-\bar{u}+\gamma(y-\bar{y})) \bar{\mu}_{2} d s(x) & \geq \delta_{2}\left\|u_{1}-\bar{u}+\gamma S^{\prime}(\bar{u})\left(u_{1}-\bar{u}\right)\right\|_{L^{1}\left(A_{2}\right)} \\
& =\delta_{2}\left\|w+\gamma S^{\prime} w\right\|_{L^{1}\left(A_{2}\right)}
\end{aligned}
$$

with $w:=u_{1}-\bar{u}$. Notice, that $w=0$ on $\Gamma \backslash A_{2}$. Moreover we set $v=G^{\prime} w$ and

$$
z= \begin{cases}0 & \text { on } \Gamma \backslash A_{2} \\ w+\left.\gamma v\right|_{\Gamma} & \text { on } A_{2}\end{cases}
$$

By this definition, we have

$$
\left\|w+\gamma S^{\prime} w\right\|_{L^{1}\left(A_{2}\right)}=\|z\|_{L^{1}\left(A_{2}\right)}
$$

and therefore

$$
\int_{\Gamma}(u-\bar{u}+\gamma(y-\bar{y})) \bar{\mu}_{2} d s(x) \geq \delta_{2}\|z\|_{L^{1}\left(A_{2}\right)} .
$$

Then we find

$$
\begin{aligned}
A v+v & =0 & & \text { in } \Omega \\
\partial_{n_{A}} v-\bar{b}_{y} v & =\bar{b}_{u} w & & \text { on } \Gamma .
\end{aligned}
$$

On $A_{2}$ we have

$$
\bar{b}_{u} w=\bar{b}_{u}\left(z-\left.\gamma v\right|_{\Gamma}\right)=\bar{b}_{u} z-\left.\chi_{A_{2}} \bar{b}_{u} \gamma v\right|_{\Gamma} .
$$

Because of $z=w=0$ on $\Gamma \backslash A_{2}$, this equation is also correct on $\Gamma \backslash A_{2}$ and consequently it holds

$$
\begin{aligned}
A v+v & =0 & & \text { in } \Omega \\
\partial_{n_{A}} v+\left(-\bar{b}_{y}+\chi_{A_{2}} \bar{b}_{u} \gamma\right) v & =\bar{b}_{u} z & & \text { on } \Gamma .
\end{aligned}
$$


Applying Lemma 3.3, we obtain

$$
\|v\|_{L^{1}(\Gamma)} \leq c\|z\|_{L^{1}(\Gamma)}=c\|z\|_{L^{1}\left(A_{2}\right)} .
$$

Setting $\bar{\gamma}=\|\gamma\|_{C(\Gamma)}$, we get

$$
\begin{aligned}
\|w\|_{L^{1}\left(A_{2}\right)} & =\|z-\gamma v\|_{L^{1}\left(A_{2}\right)} \\
& \leq\|z\|_{L^{1}\left(A_{2}\right)}+\bar{\gamma}\|v\|_{L^{1}\left(A_{2}\right)} \\
& \leq\|z\|_{L^{1}\left(A_{2}\right)}+\bar{\gamma} c\|z\|_{L^{1}\left(A_{2}\right)}
\end{aligned}
$$

or

$$
\|z\|_{L^{1}\left(A_{2}\right)} \geq \frac{1}{1+\bar{\gamma} c}\|w\|_{L^{1}\left(A_{2}\right)}
$$

Combining (3.26) and (3.30), we find

$$
\int_{\Gamma}(u-\bar{u}+\gamma(y-\bar{y})) \bar{\mu}_{2} d s(x) \geq \frac{\delta_{2}}{1+\bar{\gamma} c}\|w\|_{L^{1}\left(A_{2}\right)}=\frac{\delta_{2}}{1+\bar{\gamma} c}\left\|u_{1}-\bar{u}\right\|_{L^{1}\left(A_{2}\right)} .
$$

Invoking again $\|u-\bar{u}\|_{L^{\infty}(\Gamma)}<\varepsilon$ and (3.31), we obtain

$$
\begin{aligned}
\int_{A_{2}}(u-\bar{u}+\gamma(y-\bar{y})) \bar{\mu}_{2} d s(x) & \geq \frac{\delta_{2}}{1+\bar{\gamma} c}\left\|u_{1}-\bar{u}\right\|_{L^{1}\left(A_{2}\right)} \cdot \frac{\|u-\bar{u}\|_{L^{\infty}\left(A_{2}\right)}}{\varepsilon} \\
& \geq \frac{\delta_{2}}{c_{4} \varepsilon(1+\bar{\gamma} c)}\left\|u_{1}-\bar{u}\right\|_{L^{2}\left(A_{2}\right)}^{2},
\end{aligned}
$$

implying inequality $(3.21)$ with $c_{A}=\frac{1}{c_{4}(1+\bar{\gamma} c)}$.

If $A_{1} \cup A_{2}=\Gamma$, then the critical subspace contains only the function $(y, u)=(0,0)$. Then the assumptions of Theorem 5.3 are trivially fulfilled. In this case, (3.20) and (3.21) imply the so-called first-order sufficient optimality conditions.

4. Second-order sufficient optimality condition. Here, we outline the proof of the sufficiency Theorem 5.3. This part is very similar to the discussion in [18]. Nevertheless, we confine ourselves to the main arguments but shorten the proof for convenience of the reader.

We select from an arbitrary admissible control $u$ in a sufficiently small $L^{\infty}$ neighborhood of $\bar{u}$ and have to show that $F(y, u) \geq F(\bar{y}, \bar{u})$. Let us introduce the increments $\delta u:=u-\bar{u}$ and $\delta y:=G^{\prime}(\bar{u}) \delta u$. We split $\delta u=u_{0}+u_{+}$, where

$$
\begin{array}{lll}
u_{0}=0, & u_{+}=\delta u & \text { on } A_{1}, \\
u_{0}=\delta u, & u_{+}=0 & \text { on } \Gamma \backslash\left(A_{1} \cup A_{2}\right), \\
u_{0}=-\gamma S^{\prime}(\bar{u}) u_{0}, & u_{+}=\delta u-u_{0} & \text { on } A_{2} .
\end{array}
$$

Notice that $u_{0}+\gamma S^{\prime}(\bar{u}) u_{0}=0$ on $A_{2}$. This setting is justified again by Lemma 3.4: It holds

$$
u_{0}= \begin{cases}\phi & \text { on } \Gamma \backslash A_{2}, \\ \phi-\gamma S^{\prime} u_{0} & \text { on } A_{2}\end{cases}
$$

where $\phi$ is defined by

$$
\phi=\left\{\begin{array}{lc}
0 & \text { on } A_{1} \cup A_{2}, \\
\delta u & \text { on } \Gamma \backslash\left(A_{2} \cup A_{1}\right) . \\
12
\end{array}\right.
$$


The part $u_{0}$ belongs to the critical subspace, while $u_{+}$is the part of $\delta u$ that accounts for the effects of first-order sufficiency. Furthermore, we define $y_{0}:=G^{\prime} u_{0}$ and $y_{+}:=$ $G^{\prime} u_{+}$. By the linearity of $G^{\prime}$, we have $\delta y=y_{0}+y_{+}$.

Below, we estimate the difference $L\left(y, u, \bar{p}, \bar{\mu}_{1}, \bar{\mu}_{2}\right)-L\left(\bar{y}, \bar{u}, \bar{p}, \bar{\mu}_{1}, \bar{\mu}_{2}\right)$. Let us write for short $L(y, u)-L(\bar{y}, \bar{u})$, since $\left(\bar{p}, \bar{\mu}_{1}, \bar{\mu}_{2}\right)$ remains fixed in all the next considerations. We also do not explicitely indicate the point $\left(\bar{y}, \bar{u}, \bar{p}, \bar{\mu}_{1}, \bar{\mu}_{2}\right)$ where all derivatives are taken, i.e. we write $L_{u} u$ instead of $D_{u} L\left(\bar{y}, \bar{u}, \bar{p}, \bar{\mu}_{1}, \bar{\mu}_{2}\right) u$.

LEMMA 4.1. Under the assumptions of Theorem 5.3,

$$
L(y, u)-L(\bar{y}, \bar{u}) \geq \frac{\delta}{4}\left\|u_{0}\right\|_{L^{2}(\Gamma)}^{2}-\frac{c_{s}}{2}\left\|u_{+}\right\|_{L^{2}(\Gamma)}^{2}+r_{2}+\tilde{r}_{2}
$$

holds, where $r_{2}, \tilde{r}_{2}$ are second-order remainder terms with

$$
\frac{\left|r_{i}\right|}{\|u-\bar{u}\|_{L^{2}(\Gamma)}^{2}} \rightarrow 0 \quad \text { if }\|u-\bar{u}\|_{L^{\infty}(\Gamma)} \rightarrow 0
$$

Proof. Using a Taylor expansion, in view of (FON) we get

$$
\begin{aligned}
L(y, u)-L(\bar{y}, \bar{u})= & L_{u}[u-\bar{u}]+L_{y}[y-\bar{y}]+\frac{1}{2}\left(L_{u u}[u-\bar{u}]^{2}\right. \\
& \left.+2 L_{u y}[u-\bar{u}, y-\bar{y}]+L_{y y}[y-\bar{y}]^{2}\right)+r_{2} \\
= & \frac{1}{2}\left(L_{u u}[u-\bar{u}]^{2}+2 L_{u y}[u-\bar{u}, y-\bar{y}]+L_{y y}[y-\bar{y}]^{2}\right)+r_{2} .
\end{aligned}
$$

The following property of the remainder is known

$$
\frac{\left|r_{2}(\bar{u}, h)\right|}{\|h\|_{L^{2}(\Gamma)}^{2}} \rightarrow 0 \quad \text { as }\|h\|_{L^{\infty}(\Gamma)} \rightarrow 0 .
$$

For the proof we refer to [22]. According to the notation of Lemma 3.4, we get $y-\bar{y}=\delta y+r_{1}$. Replacing $y-\bar{y}$ by $\delta y$ in (4.2), another second order remainder term is needed

$$
\begin{aligned}
\tilde{r}_{2}:= & \frac{1}{2}\left(L_{u u}[u-\bar{u}]^{2}+2 L_{u y}[u-\bar{u}, y-\bar{y}]+L_{y y}[y-\bar{y}]^{2}\right) \\
& -\frac{1}{2}\left(L_{u u}[\delta u]^{2}+2 L_{u y}[\delta u, \delta y]+L_{y y}[\delta y]^{2}\right) .
\end{aligned}
$$

It is easy to show that

$$
\frac{\left|\tilde{r}_{2}\right|}{\|u-\bar{u}\|_{L^{2}(\Gamma)}^{2}} \rightarrow 0 \quad \text { as }\|u-\bar{u}\|_{L^{\infty}(\Gamma)} \rightarrow 0 .
$$

With these notations, (4.2) admits the form

$$
L(y, u)-L(\bar{y}, \bar{u})=\frac{1}{2}\left(L_{u u}[\delta u]^{2}+2 L_{u y}[\delta u, \delta y]+L_{y y}[\delta y]^{2}\right)+r_{2}+\tilde{r}_{2}
$$

We continue by splitting the Lagrange functional in terms of $u_{0}$ and $u_{+}$,

$$
\begin{aligned}
L_{u u}[\delta u]^{2}+2 L_{u y}[\delta u, \delta y]+L_{y y}[\delta y]^{2}= & L_{u u}\left[u_{0}\right]^{2}+2 L_{u y}\left[u_{0}, y_{0}\right]+L_{y y}\left[y_{0}\right]^{2} \\
& +L_{u u}\left[u_{+}\right]^{2}+2 L_{u y}\left[u_{+}, y_{+}\right]+L_{y y}\left[y_{+}\right]^{2} \\
& +2 L_{u u}\left[u_{0}, u_{+}\right]+2 L_{u y}\left[u_{0}, y_{+}\right] \\
& +2 L_{u y}\left[u_{+}, y_{0}\right]+2 L_{y y}\left[y_{0}, y_{+}\right] .
\end{aligned}
$$


As $u_{0}$ belongs to the critical subspace, the second-order condition (SSC) yields

$$
L^{\prime \prime}\left[u_{0}, y_{0}\right]^{2}=L_{u u}\left[u_{0}\right]^{2}+2 L_{u y}\left[u_{0}, y_{0}\right]+L_{y y}\left[y_{0}\right]^{2} \geq \delta\left\|u_{0}\right\|_{L^{2}(\Gamma)}^{2} .
$$

The other terms are easily estimated by $\left\|y_{0}\right\|_{L^{2}(\Gamma)}^{2} \leq\left\|S^{\prime}\right\|^{2}\left\|u_{0}\right\|_{L^{2}(\Gamma)}^{2},\left\|y_{+}\right\|_{L^{2}(\Gamma)}^{2} \leq$ $\left\|S^{\prime}\right\|^{2}\left\|u_{+}\right\|_{L^{2}(\Gamma)}^{2}$, and by means of Young's inequality,

$$
\begin{aligned}
\mid L_{u u}\left[u_{+}\right]^{2}+2 L_{u y}\left[u_{+}, y_{+}\right]+L_{y y}\left[y_{+}\right]^{2} & \\
& +2 L_{u u}\left[u_{0}, u_{+}\right]+2 L_{u y}\left[u_{0}, y_{+}\right] \\
& +2 L_{u y}\left[u_{+}, y_{0}\right]+2 L_{y y}\left[y_{0}, y_{+}\right] \mid \leq \frac{\delta}{2}\left\|u_{0}\right\|_{L^{2}(\Gamma)}^{2}+c_{s}\left\|u_{+}\right\|_{L^{2}(\Gamma)}^{2} .
\end{aligned}
$$

In this setting, $c_{s}$ is a certain (large) constant. Combining the last two results, we arrive at

$$
L_{u u}[\delta u]^{2}+2 L_{u y}[\delta u, \delta y]+L_{y y}[\delta y]^{2} \geq \frac{\delta}{2}\left\|u_{0}\right\|_{L^{2}(\Gamma)}^{2}-c_{s}\left\|u_{+}\right\|_{L^{2}(\Gamma)}^{2} .
$$

Returning to (4.3), we end up with

$$
L(y, u)-L(\bar{y}, \bar{u}) \geq \frac{\delta}{4}\left\|u_{0}\right\|_{L^{2}(\Gamma)}^{2}-\frac{c_{s}}{2}\left\|u_{+}\right\|_{L^{2}(\Gamma)}^{2}+r_{2}+\tilde{r}_{2},
$$

which is exactly the assertion.

In the next lemma, the term $\left\|u_{+}\right\|_{L^{2}(\Gamma)}^{2}$ in (4.1) is estimated.

LEMmA 4.2. Under the assumptions of Theorem 5.3,

$$
\left(\frac{c_{s}}{2}+\frac{\delta}{4}\right)\left\|u_{+}\right\|_{L^{2}(\Gamma)}^{2} \leq c_{5}\left\|u_{1}-\bar{u}\right\|_{L^{2}\left(A_{2}\right)}^{2}+c_{6}\left\|r_{1}\right\|_{L^{2}(\Gamma)}^{2}+c_{7}\|u-\bar{u}\|_{L^{2}\left(A_{1}\right)}^{2}
$$

holds with certain positive constants $c_{5}, c_{6}$, and $c_{7}$.

Proof. First, we get on $A_{1}$

$$
\left\|u_{+}\right\|_{L^{2}\left(A_{1}\right)}=\|\delta u\|_{L^{2}\left(A_{1}\right)}=\|u-\bar{u}\|_{L^{2}\left(A_{1}\right)} .
$$

On the whole set $\Gamma$ we have

$$
u_{+}+u_{0}=\delta u=u-\bar{u} .
$$

We apply the operator $I+\gamma S^{\prime}$ to this equation and consider the image only on the set $A_{2}$. Using $u_{0}=-\gamma S^{\prime} u_{0}$ on $A_{2}$, we find

$$
u_{+}+\gamma S^{\prime} u_{+}=u+\gamma S^{\prime} u-\left(\bar{u}+\gamma S^{\prime} \bar{u}\right) \text { on } A_{2} .
$$

Now, $u$ is again replaced by $u_{1}+u_{2}$, see (3.18), to obtain on $A_{2}$

$$
u_{+}+\gamma S^{\prime} u_{+}=u_{1}+\gamma S^{\prime} u_{1}+u_{2}+\gamma S^{\prime} u_{2}-\left(\bar{u}-\gamma S^{\prime} \bar{u}\right) .
$$

On $A_{2}$, by definition, the equation $u_{2}+\gamma S^{\prime} u_{2}=-r_{1}$ is satisfied. Therefore, here we are able to continue by

$$
u_{+}+\gamma S^{\prime} u_{+}=u_{1}-\bar{u}+\left(\gamma S^{\prime}(\bar{u})\left(u_{1}-\bar{u}\right)\right)-r_{1} \text { on } A_{2} .
$$


Due to our definitions, $u_{+}=\delta u=u-\bar{u}$ holds on $A_{1}$. In addition, $u_{+}$vanishes on $\Gamma \backslash\left(A_{1} \cup A_{2}\right)$. Therefore, we find

$$
u_{+}= \begin{cases}u_{1}-\bar{u}-\gamma S^{\prime}(\bar{u})\left(u_{+}-u_{1}+\bar{u}\right)-r_{1} & \text { on } A_{2} \\ u-\bar{u} & \text { on } A_{1} \\ 0 & \text { on } \Gamma \backslash\left(A_{1} \cup A_{2}\right) .\end{cases}
$$

Again we have a construction that was investigated in Lemma 3.4. Applying (3.11), we get the inequality

$$
\left\|u_{+}\right\|_{L^{2}(\Gamma)} \leq c_{2}\|\phi\|_{L^{2}(\Gamma)}
$$

where $\phi$ is defined by

$$
\phi= \begin{cases}-r_{1}+\left(u_{1}-\bar{u}\right)+\gamma S^{\prime}(\bar{u})\left(u_{1}-\bar{u}\right) & \text { on } A_{2} \\ u-\bar{u} & \text { on } A_{1} \\ 0 & \text { on } \Gamma \backslash\left(A_{1} \cup A_{2}\right) .\end{cases}
$$

Therefore, we obtain

$$
\left\|u_{+}\right\|_{L^{2}(\Gamma)} \leq c_{2}\left(\|u-\bar{u}\|_{L^{2}\left(A_{1}\right)}+c_{8}\left\|u_{1}-\bar{u}\right\|_{L^{2}(\Gamma)}+\left\|r_{1}\right\|_{L^{2}\left(A_{2}\right)}\right),
$$

where the positive constant $c_{8}$ is related to $\left\|S^{\prime}\right\|$. Using $\left\|u_{1}-\bar{u}\right\|_{L^{2}(\Gamma)}=\left\|u_{1}-\bar{u}\right\|_{L^{2}\left(A_{2}\right)}$,

$$
\left\|u_{+}\right\|_{L^{2}(\Gamma)} \leq c_{9}\left\|u_{1}-\bar{u}\right\|_{L^{2}\left(A_{2}\right)}+c_{2}\left\|r_{1}\right\|_{L^{2}\left(A_{2}\right)}+c_{2}\|u-\bar{u}\|_{L^{2}\left(A_{1}\right)}
$$

is found. Young's inequality yields

$$
\left\|u_{+}\right\|_{L^{2}(\Gamma)}^{2} \leq 3 c_{9}\left\|u_{1}-\bar{u}\right\|_{L^{2}\left(A_{2}\right)}^{2}+3 c_{2}\left\|r_{1}\right\|_{L^{2}(\Gamma)}^{2}+3 c_{2}\|u-\bar{u}\|_{L^{2}\left(A_{1}\right)}^{2} .
$$

A multiplication by $\left(\frac{c_{s}}{2}+\frac{\delta}{4}\right)$

$$
\left(\frac{c_{s}}{2}+\frac{\delta}{4}\right)\left\|u_{+}\right\|_{L^{2}(\Gamma)}^{2} \leq c_{5}\left\|u_{1}-\bar{u}\right\|_{L^{2}\left(A_{2}\right)}^{2}+c_{6}\left\|r_{1}\right\|_{L^{2}(\Gamma)}^{2}+c_{7}\|u-\bar{u}\|_{L^{2}\left(A_{1}\right)}^{2}
$$

concludes the proof of the lemma.

Now we are able to prove our main result Theorem 5.3.

Proof. (Theorem 5.3) Inserting (4.4) in (4.1),

$$
\begin{aligned}
L(y, u)-L(\bar{y}, \bar{u}) \geq & \frac{\delta}{4}\left(\left\|u_{0}\right\|_{L^{2}(\Gamma)}^{2}+\left\|u_{+}\right\|_{L^{2}(\Gamma)}^{2}\right)+r_{2}+\tilde{r}_{2} \\
& -c_{7}\|u-\bar{u}\|_{L^{2}\left(A_{1}\right)}^{2}-c_{5}\left\|u_{1}-\bar{u}\right\|_{L^{2}\left(A_{2}\right)}^{2}-c_{6}\left\|r_{1}\right\|_{L^{2}(\Gamma)}^{2}
\end{aligned}
$$

is obtained. Next, we return to the objective $F$,

$$
L(y, u)-L(\bar{y}, \bar{u})=F(y, u)-F(\bar{y}, \bar{u})-\int_{\Gamma} \bar{\mu}_{1}(u-\bar{u}) d s(x)-\int_{\Gamma}(u-\bar{u}+\gamma(y-\bar{y})) \bar{\mu}_{2} d s(x) .
$$

Using Lemma 3.5 we find

$$
\begin{aligned}
F(y, u)-F(\bar{y}, \bar{u}) \geq & \frac{\delta}{4}\left(\left\|u_{0}\right\|_{L^{2}(\Gamma)}^{2}+\left\|u_{+}\right\|_{L^{2}(\Gamma)}^{2}\right)+r_{2}+\tilde{r}_{2} \\
& +\left(\frac{\delta_{1}}{\varepsilon}-c_{7}\right)\|u-\bar{u}\|_{L^{2}\left(A_{1}\right)}^{2}+\left(c_{A} \frac{\delta_{2}}{\varepsilon}-c_{5}\right)\left\|u_{1}-\bar{u}\right\|_{L^{2}\left(A_{2}\right)}^{2} \\
& -c_{6}\left\|r_{1}\right\|_{L^{2}(\Gamma)}^{2} .
\end{aligned}
$$


Next, $\|\delta u\|_{L^{2}(\Gamma)}=\left\|u_{0}+u_{+}\right\|_{L^{2}(\Gamma)}^{2} \leq 2\left\|u_{0}\right\|_{L^{2}(\Gamma)}^{2}+2\left\|u_{+}\right\|_{L^{2}(\Gamma)}^{2}$ is applied to continue by

$$
\begin{aligned}
F(y, u)-F(\bar{y}, \bar{u}) \geq & \frac{\delta}{8}\|\delta u\|_{L^{2}(\Gamma)}^{2}+r_{2}+\tilde{r}_{2} \\
& +\left(\frac{\delta_{1}}{\varepsilon}-c_{7}\right)\|u-\bar{u}\|_{L^{2}\left(A_{1}\right)}^{2}+\left(c_{A} \frac{\delta_{2}}{\varepsilon}-c_{5}\right)\left\|u_{1}-\bar{u}\right\|_{L^{2}\left(A_{2}\right)}^{2} \\
& -c_{6}\left\|r_{1}\right\|_{L^{2}(\Gamma)}^{2} .
\end{aligned}
$$

Take now $\varepsilon$ sufficiently small, such that

$$
\frac{\delta_{1}}{\varepsilon}-c_{7} \geq 0 \quad \text { and } \quad c_{A} \frac{\delta_{2}}{\varepsilon}-c_{5} \geq 0 .
$$

Then we can omit the associated terms in (4.7),

$$
F(y, u)-F(\bar{y}, \bar{u}) \geq \frac{\delta}{8}\|\delta u\|_{L^{2}(\Gamma)}^{2}+r_{2}+\tilde{r}_{2}-c_{6}\left\|r_{1}\right\|_{L^{2}(\Gamma)}^{2} .
$$

Due to the discussions during the proof, all terms of the right-hand side (except the first one) are small with respect to $\|u-\bar{u}\|_{L^{2}(\Gamma)}^{2}$. Therefore

$$
F(y, u)-F(\bar{y}, \bar{u}) \geq \frac{\delta}{16}\|u-\bar{u}\|_{L^{2}(\Gamma)}^{2}
$$

holds if $\|u-\bar{u}\|_{L^{\infty}(\Gamma)}<\varepsilon$ and $\varepsilon$ is sufficiently small. The quadratic growth condition is proven. We can choose $\delta_{s}=\delta / 16$.

5. Generalizations. In this section, we discuss weaker assumption and possible generalization. The second-order sufficient optimality condition can be weakened: Let us define the weakly active control constraints:

$$
A_{1}^{\text {weak }}:=\left\{x \in \Gamma \backslash\left(A_{1} \cup A_{2}\right): \bar{\mu}_{1}(x)>0\right\} .
$$

On $A_{1}^{\text {weak }}$ we have almost everywhere $\bar{u}(x)=0$. The control constraints imply $u(x)-$ $\bar{u}(x) \geq 0$ a.e. on $A_{1}^{\text {weak }}$ for all admissible controls $u$. Therefore, the critical subspace can be reduced by the requirement

$$
u(x) \geq 0 \quad \forall x \in A_{1}^{\text {weak }} .
$$

The situation is more complicated for the weakly active control-state constraints:

$$
A_{2}^{\text {weak }}:=\left\{x \in \Gamma \backslash\left(A_{1} \cup A_{2} \cup A_{1}^{\text {weak }}\right): \bar{\mu}_{2}(x)>0\right\} .
$$

On $A_{2}^{\text {weak }}$, we have $\bar{u}(x)+\gamma(x) \bar{y}(x)=c(x)$. Moreover, it holds $u(x)+\gamma(x) y(x) \geq c(x)$ for all admissible controls $u$ with corresponding state $y$. This implies $u-\bar{u}+\gamma(y-\bar{y}) \geq$ 0 or

$$
u-\bar{u}+\gamma(x) S^{\prime}(\bar{u})(u-\bar{u})+r_{1} \geq 0
$$

on $A_{2}^{\text {weak }}$. Now, the remainder part $r_{1}$ has to be considered in the cone associated with the weakly active control-state constraints. Therefore, we abstain from an additional requirement on $A_{2}^{\text {weak }}$. 
Corollary 5.1. Suppose that the following weakened second-order sufficient optimality condition is satisfied: The condition (SSC) is only required for elements $(y, u)$ belonging to the critical subspace (defined in Definition 2.2) fulfilling in addition

$$
u \geq 0 \quad \text { on } A_{1}^{\text {weak }}
$$

This weaker assumption ensures the result of Theorem 5.3, too.

The presented techniques can be extended to derive sufficient second-order optimality conditions for other types of optimal control problems. For instance, it applies to distributed elliptic control problems with mixed control constraints considered in $\Omega$. Moreover, it works for two-sided constraints on the control, where we minimize (1.1) subject to $(1.2)$,

$$
u_{a} \leq u(x) \leq u_{b} \quad \text { for } x \in \Gamma
$$

together mixed control-state constraints

$$
c(x) \leq u(x)+\gamma(x) y(x) \quad \text { for } x \in \Gamma \text {. }
$$

In this case, the Lagrange functional is

$$
\begin{aligned}
L\left(y, u, p, \mu_{1}, \mu_{2}, \mu_{3}\right)= & F(y, u)+\int_{\Omega}\left(\sum_{i, j=1}^{m} a_{i j} D_{j} y D_{i} p+y p\right) d x-\int_{\Gamma} b p d s(x) \\
& -\int_{\Gamma} \mu_{1}\left(u-u_{a}\right) d s(x)+\int_{\Gamma} \mu_{3}\left(u-u_{b}\right) d s(x)-\int_{\Gamma}(u+\gamma y-c) \mu_{2} d s(x) .
\end{aligned}
$$

In this definition, we tacitly assume that the Lagrange multiplier $\mu_{2}$ for the constraint (5.2) is a bounded and measurable function. In contrast to the former sections, we have not been able to show this. Then, the necessary first-order optimality conditions are

$$
(F O N B O X) \begin{cases}D_{y} L\left(\bar{y}, \bar{u}, \bar{p}, \bar{\mu}_{1}, \bar{\mu}_{2}, \bar{\mu}_{3}\right) & =0 \\ D_{u} L\left(\bar{y}, \bar{u}, \bar{p}, \bar{\mu}_{1}, \bar{\mu}_{2}, \bar{\mu}_{3}\right) & =0 \\ \text { and for almost all } x \in \Gamma & \geq 0 \\ \bar{\mu}_{1}(x) & \geq 0 \\ \bar{\mu}_{3}(x) & \geq 0 \\ \bar{\mu}_{2}(x) & =0 \\ \left(\bar{u}(x)-u_{a}\right) \bar{\mu}_{1}(x) & =0 \\ \left(\bar{u}(x)-u_{b}\right) \bar{\mu}_{3}(x) & =0 . \\ (\bar{u}(x)+\gamma(x) \bar{y}(x)-c(x)) \bar{\mu}_{2}(x) & =\end{cases}
$$

Definition 5.2. The strongly active sets for problem (1.1), (1.2), (5.1), (5.2) are

$$
\begin{aligned}
& A_{1}\left(\delta_{1}\right):=\left\{x \in \Gamma: \bar{\mu}_{1}(x) \geq \delta_{1}\right\} \\
& A_{3}\left(\delta_{3}\right):=\left\{x \in \Gamma: \bar{\mu}_{3}(x) \geq \delta_{3}\right\} \\
& A_{2}\left(\delta_{2}\right):=\left\{x \in \Gamma \backslash\left(A_{1}\left(\delta_{1}\right) \cup A_{3}\left(\delta_{3}\right)\right): \bar{\mu}_{2}(x) \geq \delta_{2}\right\} .
\end{aligned}
$$


A pair $(y, u) \in C(\bar{\Omega}) \times L^{\infty}(\Gamma)$ belongs to the critical subspace, if

$$
\begin{aligned}
u=0 & & \text { on } A_{1}\left(\delta_{1}\right) \cup A_{3}\left(\delta_{3}\right), \\
u+\left.\gamma y\right|_{\Gamma}=0 & & \text { on } A_{2}\left(\delta_{2}\right),
\end{aligned}
$$

and

$$
\begin{aligned}
A y+y & =0 & & \text { in } \Omega \\
\partial_{n_{A}} y-\bar{b}_{y} y & =\bar{b}_{u} u & & \text { in } \Gamma .
\end{aligned}
$$

Again, (5.8) implies $\left.y\right|_{\Gamma}=S^{\prime}(\bar{u}) u$.

(SSCBOX): There exist positive numbers $\delta, \delta_{1}, \delta_{2}, \delta_{3}$ such that the definiteness condition

$$
L_{(u, y)}^{\prime \prime}\left(\bar{y}, \bar{u}, \bar{p}, \bar{\mu}_{1}, \bar{\mu}_{2}\right)\left[h_{y}, h_{u}\right]^{2} \geq \delta\left\|h_{u}\right\|_{L^{2}(\Gamma)}^{2}
$$

holds true for all $\left(h_{y}, h_{u}\right)$ belonging to the critical subspace defined upon $\delta_{1}, \delta_{2}, \delta_{3}$.

THEOREM 5.3. (Second-order sufficiency for box constraints and mixed constraints) Assume that $\left(\bar{y}, \bar{u}, \bar{p}, \bar{\mu}_{1}, \bar{\mu}_{2}, \overline{\mu_{3}}\right)$ fulfils the first-order optimality system (FON$B O X$ ) and the regularity condition $(R)$ is satisfied. If the second-order condition (SS$C B O X)$ is satisfied, then there exist $\delta_{s}>0$ and $\varepsilon>0$ such that the quadratic growth condition

$$
F(y, u)-F(\bar{y}, \bar{u}) \geq \delta_{s}\|u-\bar{u}\|_{L^{2}(\Gamma)}^{2}
$$

holds for all admissible pairs $(y, u)$ with $\|u-\bar{u}\|_{L^{\infty}(\Gamma)}<\varepsilon$. Therefore, $\bar{u}$ is a locally optimal control in the norm of $L^{\infty}(\Gamma)$.

This result can be shown along the lines of the Sections 3 and 4 with minor modifications: For the upper control constraint, we find another estimate of the type (3.20). In Section $4, A_{1}$ has to be replaced by $A_{1} \cup A_{3}$ and $\delta_{1}$ by $\min \left(\delta_{1}, \delta_{3}\right)$.

\section{REFERENCES}

[1] J.-J. Alibert And J.-P. Raymond, Boundary control of semilinear elliptic equations with discontinuous leading coefficients and unbounded controls, Numer. Funct. Anal. and Optimization, 3\&4 (1997), pp. 235-250.

[2] N. ARAda AND J. P. RAYMONd, Optimal control problems with mixed control-state constraints, SIAM J. Control, 39 (2000), pp. 1391-1407.

[3] M. Bergounioux And F. TröLtzsch, Optimal control of semilinear parabolic equations with state-constraints of bottleneck type, ESAIM, Control, Optimisation and Calculus of Variations, 4 (1999), pp. 595-608.

[4] J. F. Bonnans, Second-order analysis for control constrained optimal control problems of semilinear elliptic equations, Appl. Math. Optim., 38 (1998), pp. 303-325.

[5] E. CASAS, Control of an elliptic problem with pointwise state constraints, SIAM J. Control and Optimization, 4 (1986), pp. 1309-1322.

[6] - Boundary control of semilinear elliptic equations with pointwise state constraints, SIAM J. Control and Optimization, 31 (1993), pp. 993-1006.

[7] E. CASAS And M. Mateos, Second order sufficient optimality conditions for semilinear elliptic control problems with finitely many state constraints, SIAM J. Control and Optimization, 40 (2002), pp. 1431-1454.

[8] E. Casas AND F. Tröltzsch, Second-order necessary optimality conditions for some stateconstrained control problems of semilinear elliptic equations, Applied Math. and Optimization, 39 (1999), pp. 211-227.

[9] E. Casas, F. Tröltzsch, and A. Unger, Second order sufficient optimality conditions for a nonlinear elliptic control problem, Z. für Analysis und ihre Anwendungen (ZAA), 15 (1996), pp. 687-707. 
[10] - Second order sufficient optimality conditions for some state-constrained control problems of semilinear elliptic equations, SIAM J. Control and Optimization, 38 (2000), pp. 1369-1391.

[11] A. L. Dontchev, W. W. Hager, A. B. Poore, and B. Yang, Optimality, stability, and convergence in nonlinear control, Applied Math. and Optimization, 31 (1995), pp. 297326 .

[12] H. Goldberg AND F. TröLtzsch, Second order sufficient optimality conditions for a class of non-linear parabolic boundary control problems, SIAM J. Control and Optimization, 31 (1993), pp. 1007-1025.

[13] M. Heinkenschloss and F. Tröltzsch, Analysis of the Lagrange-SQP-Newton method for the control of a phase field equation, Control and Cybernetics, 28 (1999), pp. 178-211.

[14] H. MAURER, First and second order sufficient optimality conditions in mathematical programming and optimal control, Math. Programming Study, 14 (1981), pp. 163-177.

[15] J.-P. RAYmond And F. TRÖLtzsch, Second order sufficient optimality conditions for nonlinear parabolic control problems with state constraints, Discrete and Continuous Dynamical Systems, 6 (2000), pp. 431-450.

[16] A. RösCh AND F. TRÖLTZSCH, Existence of regular lagrange multipliers for a nonlinear elliptic optimal control problem with pointwise control-state constraints, submitted.

[17] _ Sufficient second order optimality condititions for a state-constrained optimal control problem of a weakly singular integral equation, Num. Funct. Anal. Appl., 23 (2002), pp. $173-193$.

[18] - Sufficient second order optimality conditions for a parabolic optimal control problem with pointwise control-state constraints, SIAM J. Control and Optimization, 42 (2003), pp. 138-154.

[19] G. Stampacchia, Le problème de Dirichlet pour les équations elliptiques du second ordre à coefficients discontinus, Ann. Inst. Fourier, Grenoble, 15 (1965), pp. 189-258.

[20] H. Triebel, Interpolation Theory, Function Spaces, Differential Operators, J. A. Barth Verlag, Heidelberg-Leipzig, 1995.

[21] F. TRÖLtzSCH, Regular Lagrange multipliers for control problems with mixed pointwise controlstate constraints, SIAM J. on Optimization, to appear.

[22] - Approximation of non-linear parabolic boundary control problem by the Fourier method convergence of optimal controls, Optimization, 22 (1991), pp. 83-98. 